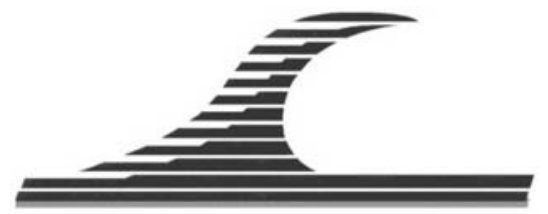

\title{
Stabilité d'un écoulement cisaillé modélisant la crème de vase
}

\author{
Alice HARANG ${ }^{1,2}$, Olivier THUAL ${ }^{1,2}$, \\ Pierre BRANCHER $^{1,2}$, Thomas BONOMETTI ${ }^{1,2}$
}

\author{
1. Université de Toulouse, INPT, UPS, IMFT, Allée Camille Soula, \\ F-31400 Toulouse, France. \\ 2. CNRS, IMFT, F-31400 Toulouse, France. \\ harang@imft.fr ; thual@imft.fr ; brancher@imft.fr ; thomas.bonometti@imft.fr
}

\section{Résumé :}

Afin d'améliorer la compréhension de la mise en suspension de la vase au fond des estuaires, une étude paramétrique de la stabilité d'un écoulement bidimensionnel cisaillé modélisant deux couches fluides miscibles de masse volumique et de viscosité dynamique différentes. Le code de simulation numérique directe JADIM de l'IMFT (Institut de Mécanique des Fluides de Toulouse) est utilisé pour calculer l'évolution temporelle de ces écoulements. Le modèle est initialisé à l'aide de profils verticaux continus de type $\operatorname{erf}(z)$ pour toutes les grandeurs considérées. Cette étude est ensuite complétée par une étude de stabilité linéaire réalisée avec le code LiSa développé à l'IMFT. Un nombre de Richardson critique proche de 0,25 est observé. De forts rapports de viscosité entre les deux fluides modifient les caractéristiques du mode le plus instable mais aussi la localisation de cette instabilité dans l'interface. Cette étude de stabilité est de nature à déboucher sur de nouvelles paramétrisations pour la modélisation réaliste des estuaires.

Soumis le 12 novembre 2010, accepté le 7 décembre 2010, en ligne le 22 décembre 2010.

La seule version examinée est celle écrite en français. La ou les autres versions n'étant pas examinées par le comité de rédaction de la revue, sont donc publiées sous l'entière responsabilité du ou des auteurs.

A TRANSLATED VERSION IN ENGLISH IS AVAILABLE ONLINE

Pour citer cet article :

HARANG A., THUAL O., BRANCHER P., BONOMETTI T. (2010). Stabilité d'un écoulement cisaillé modélisant la crème de vase. Revue Paralia, Vol. 3, pp 8.1-8.12.

DOI:10.5150/revue-paralia.2010.008 (disponible en ligne - http://www.paralia.fr - available online) 


\section{Introduction}

Les modèles numériques des estuaires tels que, par exemple, TELEMAC_3D ou SIAM$3 \mathrm{D}$, sont utilisés pour de nombreuses applications comme la gestion de la pollution ou les aménagements côtiers. Pour ce qui concerne la mise en suspension au niveau du fond de l'estuaire, de nombreux modèles reposent sur les paramétrisations proposées par PARTHENIADES (1965) ou par PARCHURE \& METHA (1985). Cependant, ces modèles peuvent être améliorés par une étude plus approfondie de l'interface au niveau de la crème de vase comme le propose LE NORMAND (1995) après implémentation de ces modèles dans le code TELEMAC_3D. Dans cet esprit, nous proposons ici une étude bidimensionnelle de la stabilité de cette interface. La stabilité et le mélange d'écoulements cisaillés et stratifiés ont été étudiés par CAULFIELD \& PELTIER (2000). ERN et al. (2003) ont étudié les écoulements cisaillés et stratifiés en viscosité. KRANENBURG \& WINTERWERP (1997) proposent un modèle, validé expérimentalement, de mise en suspension de la crème de vase par la turbulence créée par le vent ou advectée par l'écoulement. Comme expliqué dans PHAM VAN BANG et al. (2007), la crème de vase a un comportement très complexe, thixotrope, évoluant en fonction du temps et des forçages, ce qui rend sa modélisation très difficile. Pour la présente étude, nous choisissons une modélisation de type fluide newtonien miscible avec une forte variation spatiale de la viscosité, afin de constituer une première approche de la réponse du système à une rhéologie complexe. D'après le modèle de distribution proposé par MEHTA et al. (1989), l'épaisseur du profil de cisaillement peut être plus importante que celle du profil de densité ce qui pourrait entrainer, d'après HOGG \& IVEY (2003), le développement d'instabilités de type Holmboe à l'interface eau - crème de vase. Néanmoins, nous choisissons ici une même épaisseur pour les profils de vitesse, de densité et de viscosité afin de nous concentrer sur l'étude des instabilités de type Kelvin-Helmoltz.

\section{Modélisation de la crème de vase}

\subsection{Equations du modèle}

Notre étude est basée sur le système d'équations suivant:

$$
\begin{aligned}
& \nabla \cdot \underline{u}=0, \quad \frac{d \underline{u}}{d t}=-\frac{1}{\rho} \nabla P+\underline{g}+\frac{1}{\rho} \nabla\left[\mu\left(\nabla \underline{u}+{ }^{t} \nabla \underline{u}\right)\right], \\
& \frac{\partial \Phi}{\partial t}+\nabla \cdot(\Phi \underline{u})=0, \\
& \rho=\rho_{0}(1-\Phi)+\rho_{1} \Phi, \quad \mu=\mu_{0}(1-\Phi)+\mu_{1} \Phi
\end{aligned}
$$

qui couple les équations de Navier-Stokes incompressibles (1.a), où $g$ est le vecteur gravité, à l'équation de transport (1.b) d'une fraction volumique $\Phi$ qui contrôle, à travers les relations (1.c), le mélange cinématique de particules fluides de masse 
volumique $\rho$ et de viscosité dynamique $\mu$ inhomogènes. La diffusion moléculaire des sédiments est négligée (voir DEARDORFF \& WILLIS, 1982).

Nous considérons le mélange des deux fluides suivants : l'eau, de masse volumique $\rho_{0}=1000 \mathrm{~kg} \mathrm{~m}^{-3}$ et de viscosité dynamique $\mu_{0}=10^{-3} \mathrm{~Pa} \mathrm{~s}$, et la crème de vase, de masse volumique $\rho_{1}=1360 \mathrm{~kg} \mathrm{~m}^{-3}$ (MEHTA et al. 1989) et de viscosité dynamique $\mu_{1}=1 \mathrm{~Pa} \mathrm{~s}$. Ces deux entités sont ensuite mélangées et suivies par le taux de présence de crème de vase dans le fluide, $\Phi$. Ainsi, dans ces simulations, la masse volumique et la viscosité sont fonctions linéaires du taux de présence et ont donc des évolutions parallèles.

\subsection{Configuration de base}

Afin de modéliser les profils verticaux des grandeurs étudiées au voisinage de l'interface entre la vase et l'eau, nous considérons la famille des fonctions $F(\lambda, Z)$ définies par:

$$
F(\lambda, Z)= \begin{cases}\lambda\left[1+\operatorname{erf}\left(\frac{Z}{\lambda}\right)\right] & \text { si } \quad Z \leq 0, \\ 1-(1-\lambda)\left[1-\operatorname{erf}\left(\frac{Z}{1-\lambda}\right)\right] & \text { si } \quad Z \geq 0,\end{cases}
$$

où $Z$ est la coordonnée verticale adimensionnelle spécifiée ensuite, dont l'origine est situé à l'interface, le paramètre $\lambda$ contrôle l'asymétrie des domaines situés de part et d'autre du point d'inflexion, avec continuité du profil et de sa dérivée autour de ce point. Les caractéristiques de cette famille de profils sont décrites par la figure 1. La fonction $\operatorname{erf}(z)$ a été choisie comme fonction de base, de manière empirique, pour modéliser qualitativement un cisaillement simple. Dans certains cas, ce profil peut être vu comme décrivant une réponse transitoire à un gradient imposé en présence de diffusion turbulente.

On considère alors les profils verticaux de vitesse horizontale $u(z)$, de masse volumique $\rho(z)$ et de viscosité dynamique $\mu(z)$ donnés par les relations :

$$
\begin{aligned}
& u(z)=U_{1} F\left(\lambda, \frac{z-h}{\delta_{u}}\right), \\
& \rho(z)=\left(\rho_{0}-\rho_{1}\right) F\left(\lambda, \frac{z-h}{\delta_{\rho}}\right)+\rho_{1}, \quad \mu(z)=\left(\mu_{0}-\mu_{1}\right) F\left(\lambda, \frac{z-h}{\delta_{\mu}}\right)+\mu_{1},
\end{aligned}
$$

où $\delta_{u}, \delta_{\rho}$ et $\delta_{\mu}$ sont des épaisseurs respectivement associées aux profils $u(z), \rho(z)$ et $\mu(z)$. Nous considérons ici le cas où ces trois épaisseurs sont égales à $\delta=0,3 \mathrm{~m}$. Les frontières du domaine de la simulation étant définies par les équations $z=0$ et $z=3 h$, le choix d'une hauteur $h=1 \mathrm{~m}$ permet de les situer suffisamment loin de l'interface d'équation $z=h$. D'autre part, nous avons choisi $\lambda=0,1$, en considérant que la diffusion supposée plus importante dans l'eau que dans la vase. Nous obtenons alors les profils présentés sur la figure 2 . 


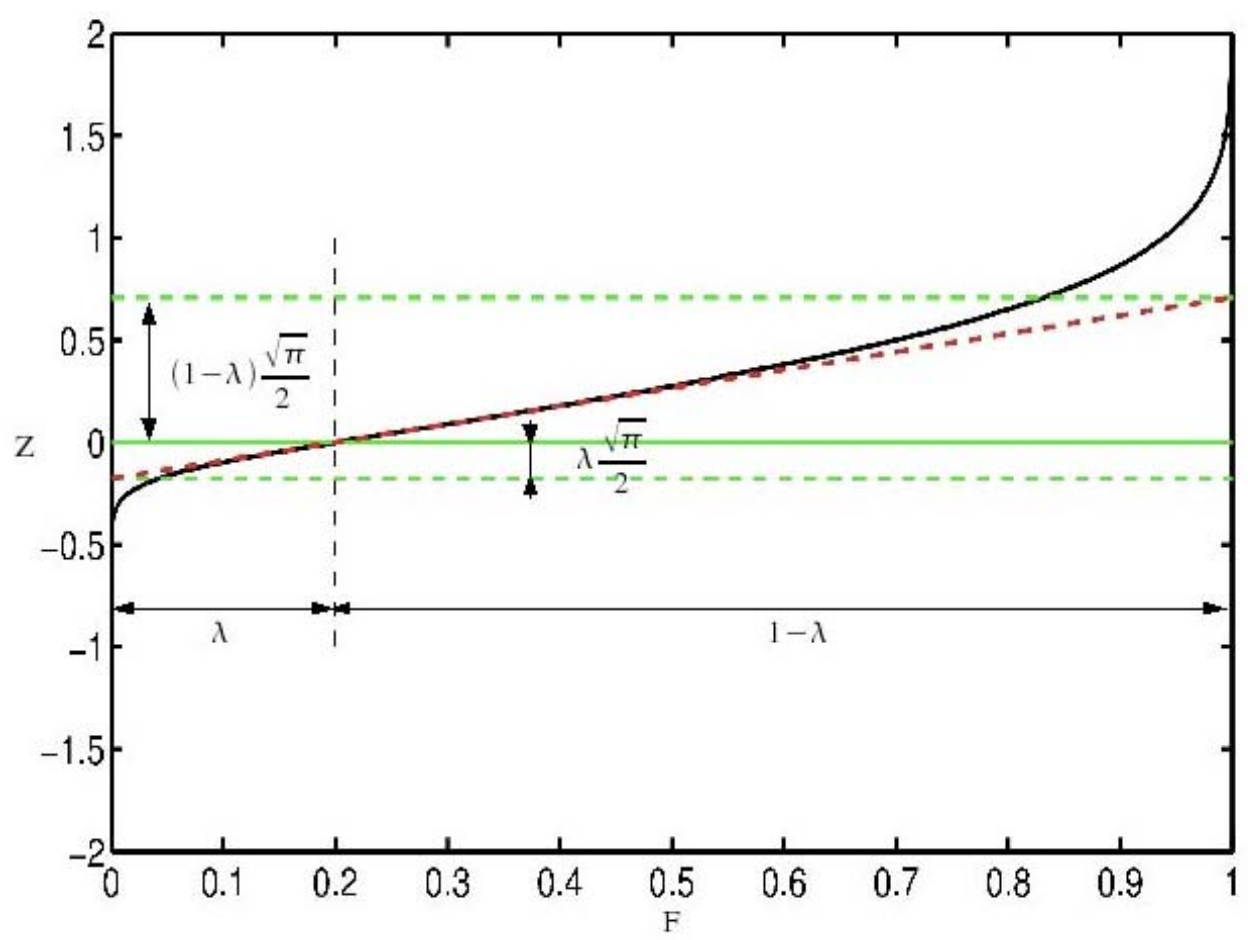

Figure 1. Représentation du profil en $Z$ de la fonction $F(\lambda, Z)$ avec $\lambda=0,2$.
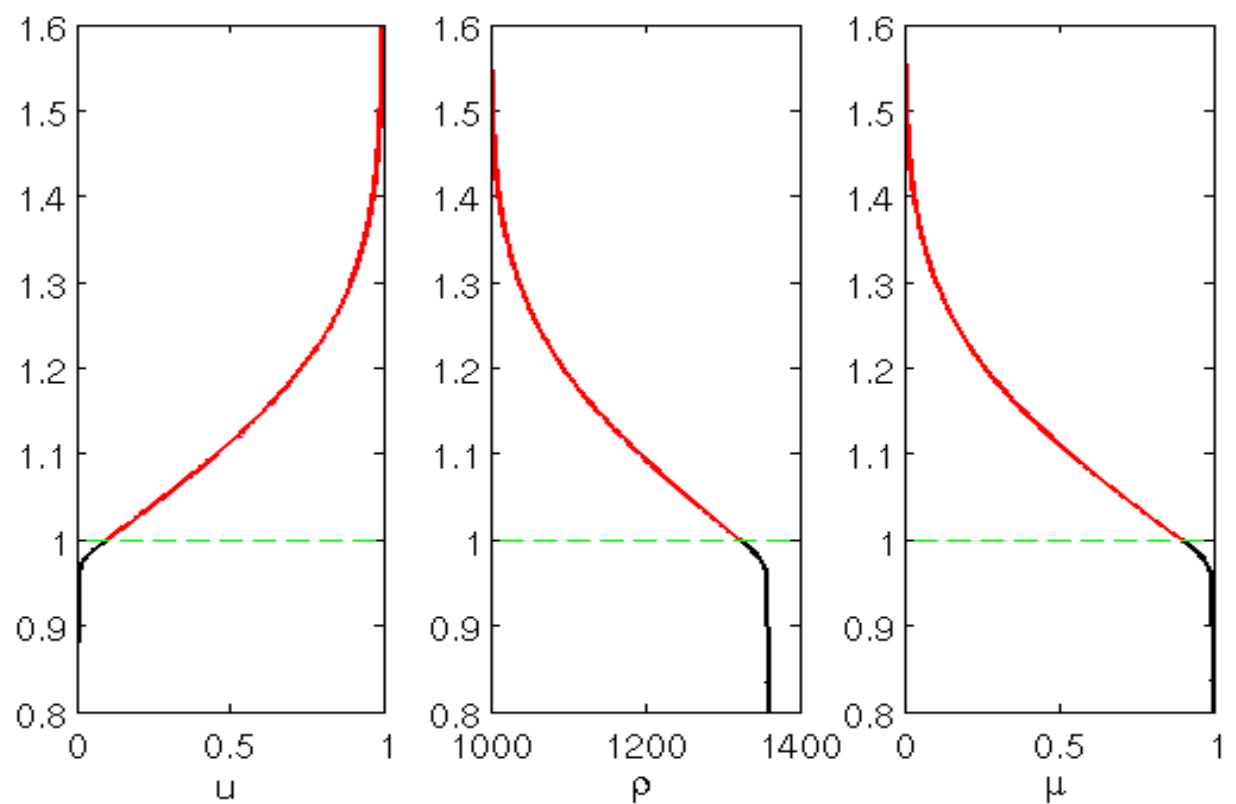

Figure 2. Profils initiaux $u(z), \rho(z)$ et $\mu(z)$ sur une portion $z \in[0,8 \mathrm{~m}, 1,6 \mathrm{~m}] d u$ domaine $z \in[0,3 \mathrm{~h}]$, avec $h=1 \mathrm{~m}, \delta=0,3 \mathrm{~m}$ et $\lambda=0,1$. 


\subsection{Paramètres de contrôle}

Nous nous plaçons tout d'abord dans des configurations où le nombre d'Atwood $A t=\left(\rho_{1}-\rho_{0}\right) /\left(\rho_{1}+\rho_{0}\right)$ est de l'ordre de 0,15 . Les trois autres nombres adimensionnels pertinents pour ce problème sont définis par :

$$
R e=\frac{U_{0} \delta}{\mu_{0} / \rho_{0}}, \quad R i=\frac{g}{\rho_{h}} \frac{\left(\rho_{0}-\rho_{1}\right) \delta \sqrt{\pi}}{2 U_{0}^{2}} \quad \text { et } \quad W=\log _{10}\left(\frac{\mu_{1}}{\mu_{0}}\right),
$$

Où $\rho_{h}=\rho(h)=\left(\rho_{0}-\rho_{1}\right) \lambda+\rho_{1}$ est la masse volumique à l'interface, le nombre de Reynolds $R e$ est basé sur la viscosité de l'eau, le nombre de Richardson $R i$ est basé sur le maximum du gradient de masse volumique situé à l'interface $z=h$, et où nous avons choisi d'exprimer le rapport des viscosités entre la vase et l'eau par son logarithme décimal $W$.

\subsection{Description des programmes de DNS et d'analyse de stabilité linéaire utilisés}

Le code de simulation numérique directe (DNS) développé au sein de l'IMFT (Institut de Mécanique des Fluides de Toulouse), JADIM, résout les équations de Navier-Stokes pour des fluides incompressibles pouvant comporter différentes phases. Les équations sont écrites en variables primitives (vitesse, pression) et la méthode des volumes finis est utilisée. Les schémas utilisés confèrent au code une précision d'ordre 2 en espace et en temps grâce à un avancement en temps de type Runge-Kutta d'ordre 3 et une résolution des termes diffusifs semi-implicite de type Crank-Nicolson. Les équations sont discrétisées en utilisant un schéma centré de deuxième ordre avec maillage décalé (CALMET \& MAGNAUDET, 1996 ; LEGENDRE, 1996). La version utilisée dans cette étude résout le système d'équations (1) et fut validée pour un gradient de viscosité par ERN et al. (2003) et pour le gradient de masse volumique par HALLEZ (2007).

En complément du code JADIM, un code de stabilité linéaire, LiSa (ANTKOWIAK \& BRANCHER, 2004; 2007) est utilisé afin d'obtenir une vision plus globale du problème. Ce code résout le système d'équations (1) linéarisé autours de l'écoulement de base (3). A partir d'un état de base de type écoulement parallèle, d'une perturbation (déterminée par son vecteur d'onde) et de paramètres numériques spécifiques, LiSa fournit le spectre des valeurs propres du système et les modes associés, en utilisant une résolution spectrale par projection sur les polynômes de Chebyshev. Nous pouvons donc obtenir pour chaque longueur d'onde choisie le mode le plus instable et son taux de croissance.

\section{Résultats}

On choisit de conserver, dans les simulations, les valeurs de masse volumique de l'eau et de la vase. Nous faisons alors varier la vitesse afin de modifier le nombre de Richardson $R i$ et le nombre de Reynolds $R e$, ainsi que la viscosité de la vase pour faire varier $W$. Pour ces simulations, le nombre de Reynolds est de l'ordre de $10^{5}$. 


\subsection{Paramètres bidimensionnels}

Dans un premier temps, des simulations bidimensionnelles, proches de l'écoulement observable au fond des estuaires sont réalisées en DNS avec le code numérique JADIM. Le choix de cette configuration bidimensionnelle a été justifié par l'exploration de configurations 3D durant lesquelles nous avons observé le développement d'instabilités dans le plan de l'écoulement. Des instabilités à composante longitudinale ne peuvent cependant pas être exclues par le Théorème de Squire, comme dans le cas d'écoulements homogènes.

Les simulations 2D sont réalisées sur un domaine de calcul périodique selon la direction de l'écoulement, de dimension $L=3,4$ m et $H=3 \mathrm{~m}$, respectivement, $340 \times 300$ mailles, et avec une condition limite d'adhérence au fond et de glissement en haut du domaine. À l'instant initial, l'écoulement parallèle est perturbé par un champ de vitesse verticale localisé autour de l'interface $z=h$ modélisé par un bruit blanc d'amplitude $w_{m}=10^{-2} \mathrm{~m} \mathrm{~s}^{-1}$.

\subsection{Développement typique d'une instabilité}

Nous présentons ici une campagne numérique de simulations 2D réalisée avec le code JADIM en faisant varier le nombre de Richardson gradient $R i$ et le rapport de viscosité $W$.

Nous observons le développement des instabilités de cisaillement de grande amplitude pour des nombres de Richardson inférieurs à une valeur critique proche de 0,25 , pour toutes les valeurs de $W$ étudiées ici.

Concernant la variation du gradient de viscosité, nous observons que, comme présenté sur les figures 3 et 4 pour un nombre de Richardson $R i=0,15$, une augmentation du gradient de viscosité (caractérisé par $W$ ) entraine un déplacement de l'instabilité de cisaillement vers le haut de l'interface, donc vers l'eau, la phase la moins visqueuse. Ce déplacement de l'instabilité a pour conséquence une diminution de la quantité de matière remise en suspension. En effet, pour un rapport de viscosité important, le mélange s'effectue entre de l'eau et de la crème de vase déjà partiellement mélangée de l'interface, de concentration intermédiaire.

\subsection{Analyse de stabilité linéaire}

Après avoir déterminé l'impact des paramètres de contrôle sur la stabilité de l'interface, une analyse de stabilité a été menée grâce au programme LiSa développé à l'IMFT. Le taux de croissance du mode le plus instable a été calculé pour une centaine de nombres d'onde adimensionnées de perturbation $k^{*}$ et présenté pour différents $W$ et pour $R i=0,15$ sur la figure 5 et pour différents $R i$ et $W=3$ sur la figure 6 . Nous avons choisi d'adimensionner le nombre d'onde par la demi-épaisseur de l'interface: $k^{*}=k \times \delta \times(\sqrt{ } \pi) / 4$; la vitesse de phase par $U_{0} / 2$; le taux de croissance est donc normalisé $\operatorname{par} \sigma^{*}=\sigma \times(\delta \times(\sqrt{ } \pi) / 2) / U_{0}$. 


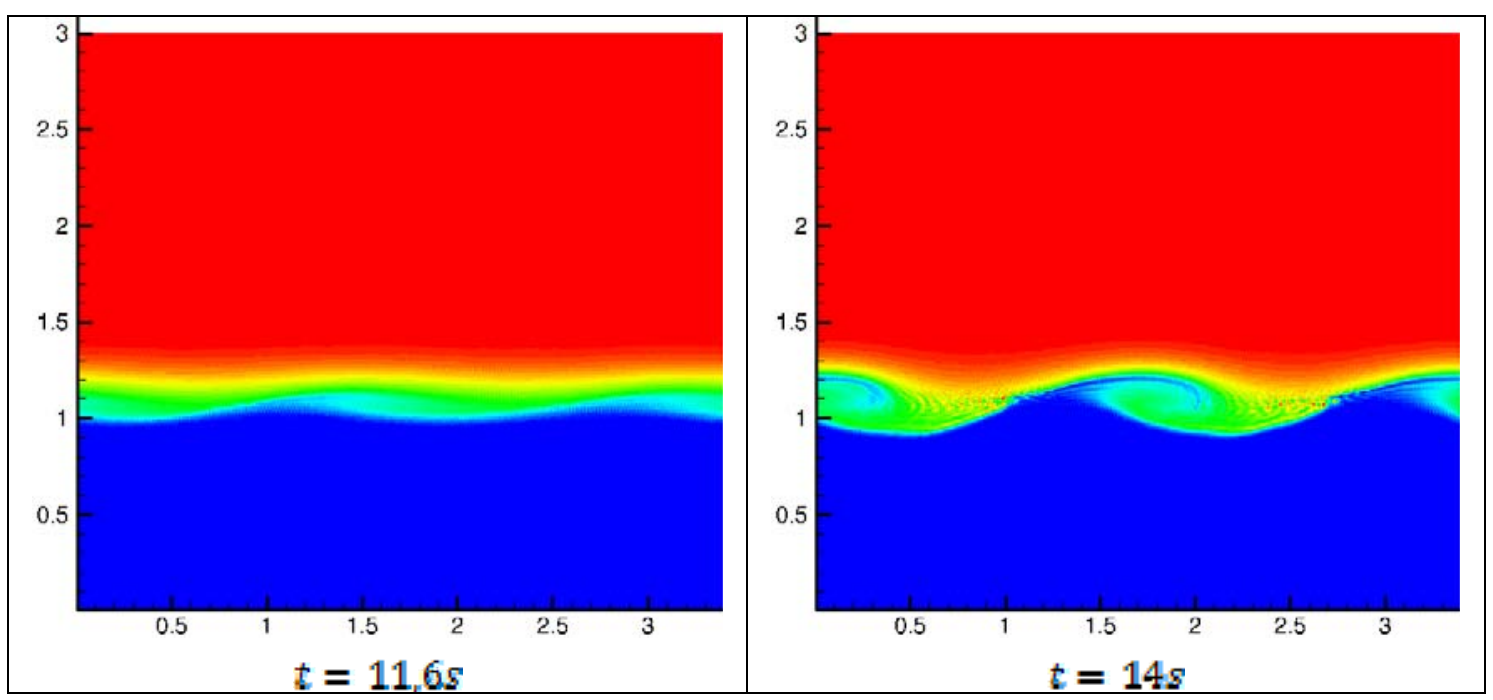

Figure 3. Configuration $R i=0,15, L=3,4 m, W=0$. Champ de masse volumique $\rho(x, z)$ à différents instants séparés de 2,3 $\mathrm{s}\left(\square: \rho_{0}=1000 \mathrm{~kg} \mathrm{~m}^{-3}, \square: \rho_{1}=1360 \mathrm{~kg} \mathrm{~m}^{-3}\right)$.

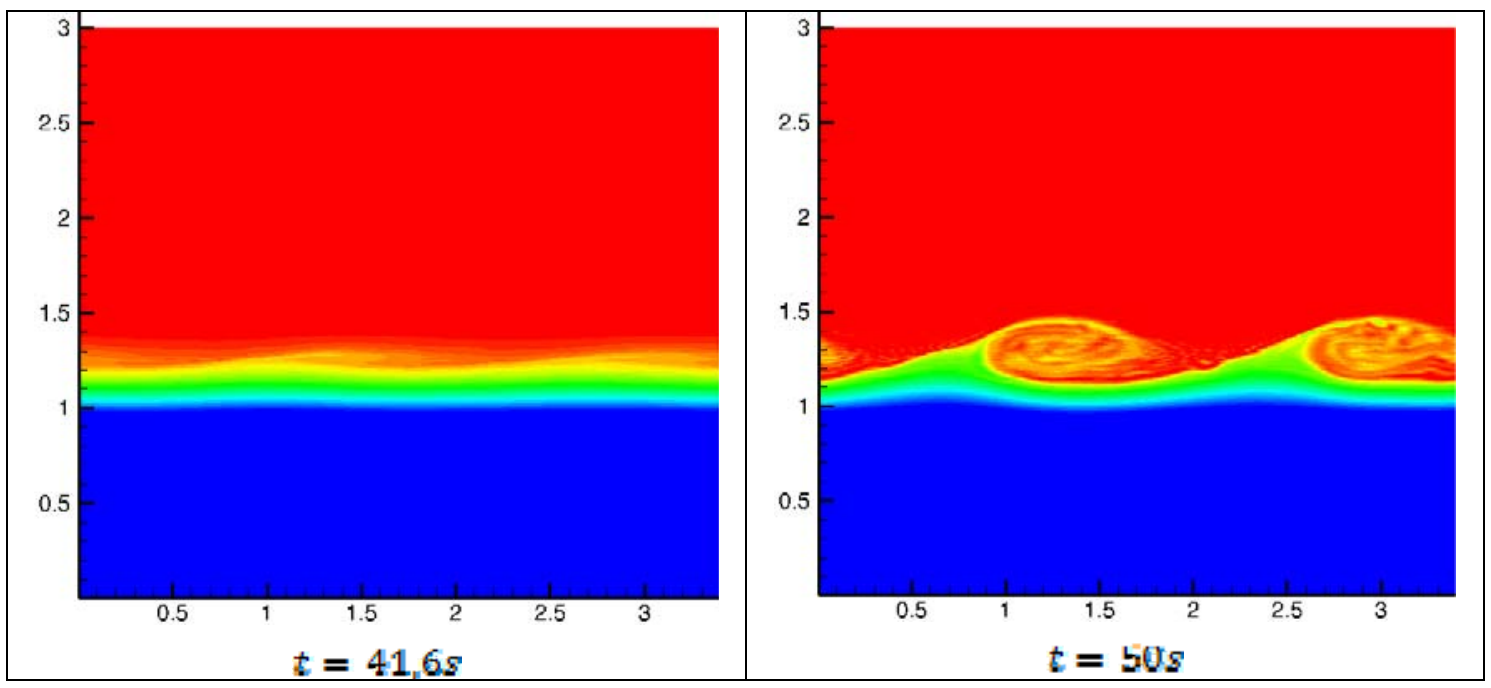

Figure 4. Configuration $R i=0,15, L=3,4 \mathrm{~m}, W=3$. Champ de masse volumique $\rho(x, z)$ à différents instants séparés de $8 \mathrm{~s}\left(\square: \rho_{0}=1000 \mathrm{~kg} \mathrm{~m}^{-3}, \square: \rho_{1}=1360 \mathrm{~kg} \mathrm{~m}^{-3}\right)$.

Nous observons sur la figure 5 des comportements similaires pour les différents rapports de viscosité testés. Cependant de légères différences sont observables pour $W=3$, correspondant à une viscosité dynamique de la crème de vase 1000 fois plus importante que celle de l'eau. D'une part, nous pouvons remarquer que le taux de croissance est plus important $(\sim 10 \%)$; d'autre part, le nombre d'onde le plus instable est différent $(0,456$ pour $W=0$ contre 0,480 pour $W=3)$. 
Cette différence de taux de croissance et de nombre d'onde le plus instable entre les différents $W$ présentés est susceptible d'avoir des conséquences sur le mélange à l'interface et donc la quantité de sédiments remis en suspension.

Sur la figure 6, nous pouvons clairement observer, lorsque $R i$ diminue, une augmentation du taux de croissance, ainsi qu'une légère augmentation du nombre d'onde le plus instable. On observe aussi pour cette configuration $(W=3)$ une subsistance d'instabilités de très faible taux de croissance au-delà du Richardson critique conventionnel situé autour de 0,25.

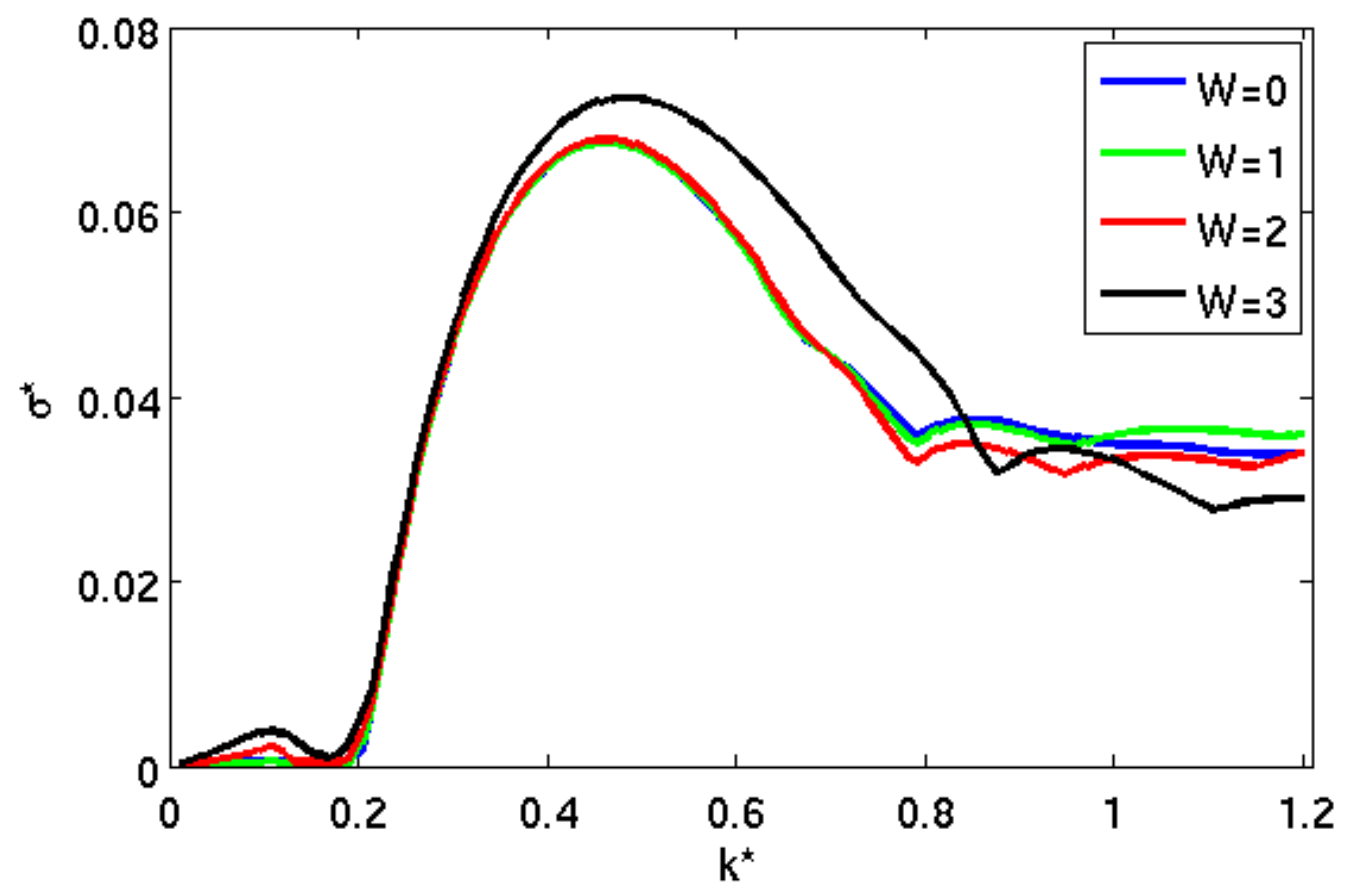

Figure 5. Représentation du taux de croissance normalisé $\sigma^{*}$ en fonction du nombre $d$ 'onde adimensionnel $k^{*}$ pour un nombre de Richardson $R i=0,15$. 


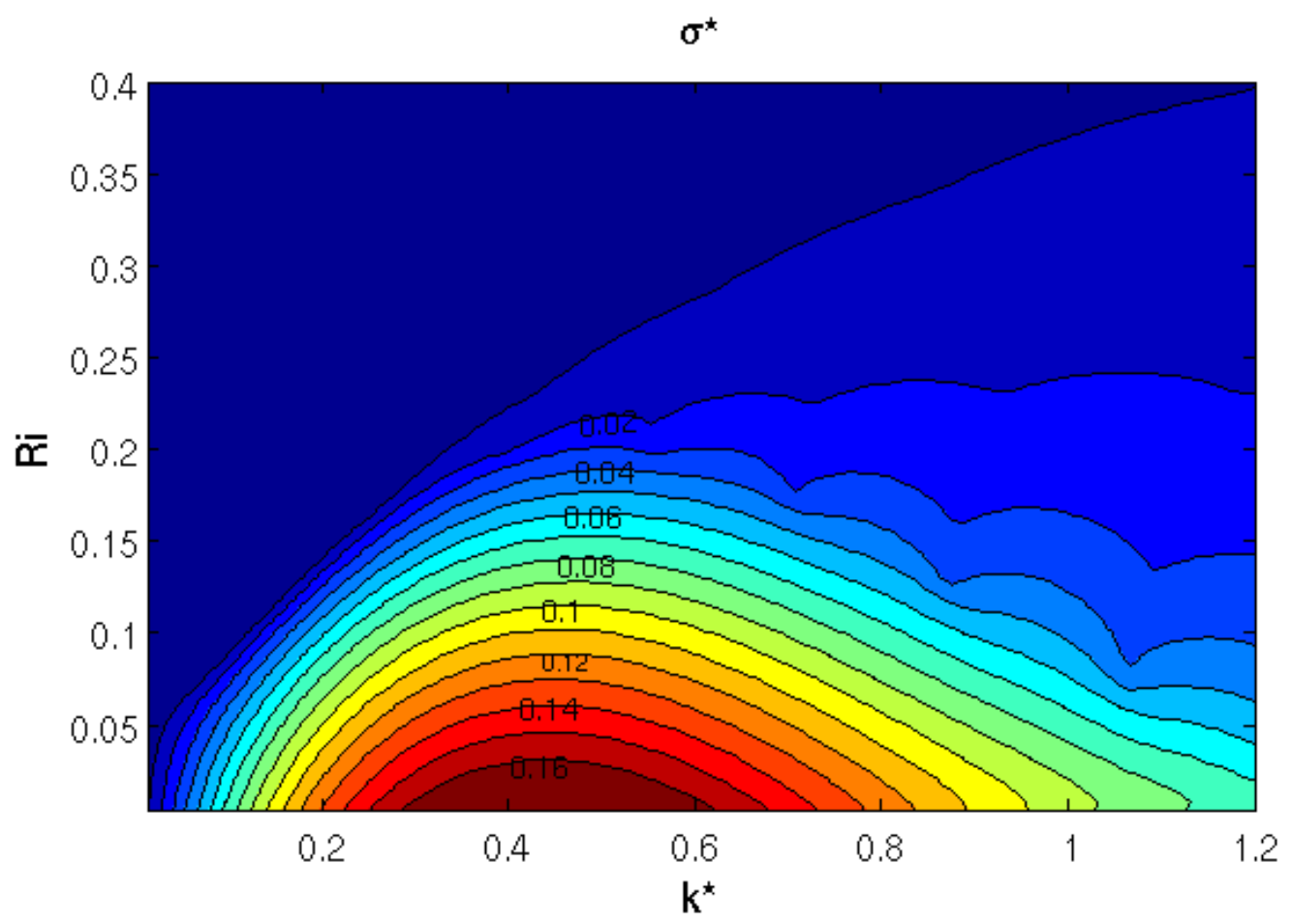

Figure 6. Isocontours du taux de croissance normalisé $\sigma^{*}$ en fonction du nombre d'onde adimensionnel $k^{*}$ et du nombre de Richardson Ri, pour $W=3$.

\section{Conclusions}

Nous avons modélisé l'interface entre la crème de vase et l'eau par l'écoulement cisaillé de fluides newtoniens miscibles présentant de fortes variations de masse volumique et de viscosité. Nous avons étudié ces écoulements en 2D pour en déduire une limite de stabilité proche de la limite théorique connue $R i=1 / 4$ pour les écoulements cisaillés stratifiés de viscosité homogène. Nos résultats montrent que le gradient de viscosité influe sur déclenchement de l'instabilité, en modifiant le nombre de Richardson critique ainsi que le nombre d'onde du mode le plus instable et sur la localisation de l'instabilité dans l'interface. Le rapport de viscosité doit donc être pris en compte dans la paramétrisation du déclenchement de mélange à l'interface par instabilité de cisaillement.

Ces résultats prédisent, au niveau de l'interface, la masse volumique d'un fluide homogène, issu du mélange d'un modèle de crème de vase et d'eau, en fonction des caractéristiques de l'écoulement. Nous avons pour objectif de traduire ensuite cette masse volumique en concentration afin de fournir une condition limite de remise en suspension. Ces résultats suggèrent aussi de nouvelles recherches, dont notamment la comparaison avec des simulations réalisées avec une rhéologie plus réaliste de la vase de type Bingham ou complexe (PHAM VAN BANG, 2007). 


\section{Remerciements}

Ce travail bénéficie du soutien financier de la Délégation Générale de DCA l'Armement (DGA) à travers une bourse de thèse attribuée à l'un des auteurs. Cette étude a pu être réalisée grâce aux moyens de calcul du CICT et du CINES.

\section{Références bibliographiques}

ANTKOWIAK A., BRANCHER P. (2004). Transient energy growth for the LambOseen vortex. Physics of Fluids, vol. 16(1), pp L1-L4. doi:10.1063/1.1626123

ANTKOWIAK A., BRANCHER P. (2007). On vortex rings around vortices: an optimal mechanism. Journal of Fluids Mechanics, vol. 578, pp 295-304. doi:10.1017/S0022112007005198

CALMET I., MAGNAUDET J. (1996). Large-eddy simulation of high-Schmidt number mass transfer in a turbulent channel flow. Physics of Fluids, vol. 9(2), pp 438-455. doi:10.1063/1.869138

CAULFIELD C.P., PELTIER W.R. (2000). The anatomy of the mixing transition in homogeneous and stratified free shear layers. Journal of Fluid Mechanics, $\mathrm{n}^{\circ} 413$, pp 1-47. doi:10.1017/S0022112000008284

DEARDORFF J.W., WILLIS G.E. (1982). Dependence of mixed-layer entrainment on shear stress and velocity jump. Journal of Fluid Mechanics, $\mathrm{n}^{\circ} 115$, pp 123-150. doi:10.1017/S0022112082000688

ERN P., CHARRU F., LUCHINI P. (2003). Stability analysis of a shear flow with strongly stratified viscosity. Journal of Fluid Mechanics, $\mathrm{n}^{\circ}$ 496, pp 295-312. doi:10.1017/S0022112003006372

HALLEZ Y. (2007). Mélange gravitationnel de fluides en géométrie confinée. Thèse, Institut National Polytechnique de Toulouse, 140 p. Disponible en ligne sur URL http://oa.imft.fr/1851/1/hallez2007.pdf

HOGG A.McC., IVEY G.N. (2003). The Kelvin-Helmoltz to Holmboe instability transition in stratified exchange flows. Journal of Fluid Mechanics, vol. 477, pp 339-362. doi:10.1017/S0022112002003397

KRANENBURG C., WINTERWERP J.C. (1997). Erosion of fluid mud layers. I: Entrainment model. Journal of Hydraulic Engineering, vol. 123, $\mathrm{n}^{\circ} 6$, pp 504-511. doi:10.1061/(ASCE)0733-9429(1997)123:6(504)

LEGENDRE D. (1996). Quelques aspects des forces hydrodynamiques et des transferts de chaleur sur une bulle sphérique. Thèse, Institut National Polytechnique de Toulouse, 266 p. Disponible sur URL http://ethesis.inp-toulouse.fr/archive/00000655/

LE NORMAND C. (1995). Modélisation numérique tridimensionnelle des processus de transport des sédiments cohésifs en environnement estuarien. Thèse, Institut National Polytechnique de Toulouse, $237 \mathrm{p}$. 
MEHTA A.J., HAYTER E.J., PARKER W.R., KRONE R.B., TEETER A.M. (1989). Cohesive sediments transport. Journal of Hydraulic Engineering, vol. 115, $\mathrm{n}^{\circ} 8$, pp 1076-1112. doi:10.1061/(ASCE)0733-9429(1989)115:8(1076)

PARCHURE T.M., MEHTA A.J. (1985). Erosion of soft cohesive sediment deposits. Journal of Hydraulic Engineering, vol. 111(10), pp 1308-1326. doi:10.1061/(ASCE)07339429(1985)111:10(1308)

PARTHENIADES E. (1965). Erosion and deposition of cohesive soils. Journal of Hydraulic Division, ASCE, vol. 91(1), pp 105-137.

PHAM VANG BANG D., OVARLEZ G., TOCQUER L. (2007). Effets de la densité et de la structuration sur les caractéristiques rhéologiques de la vase. La Houille Blanche, pp 85-93. doi:10.1051/lhb:2007023 


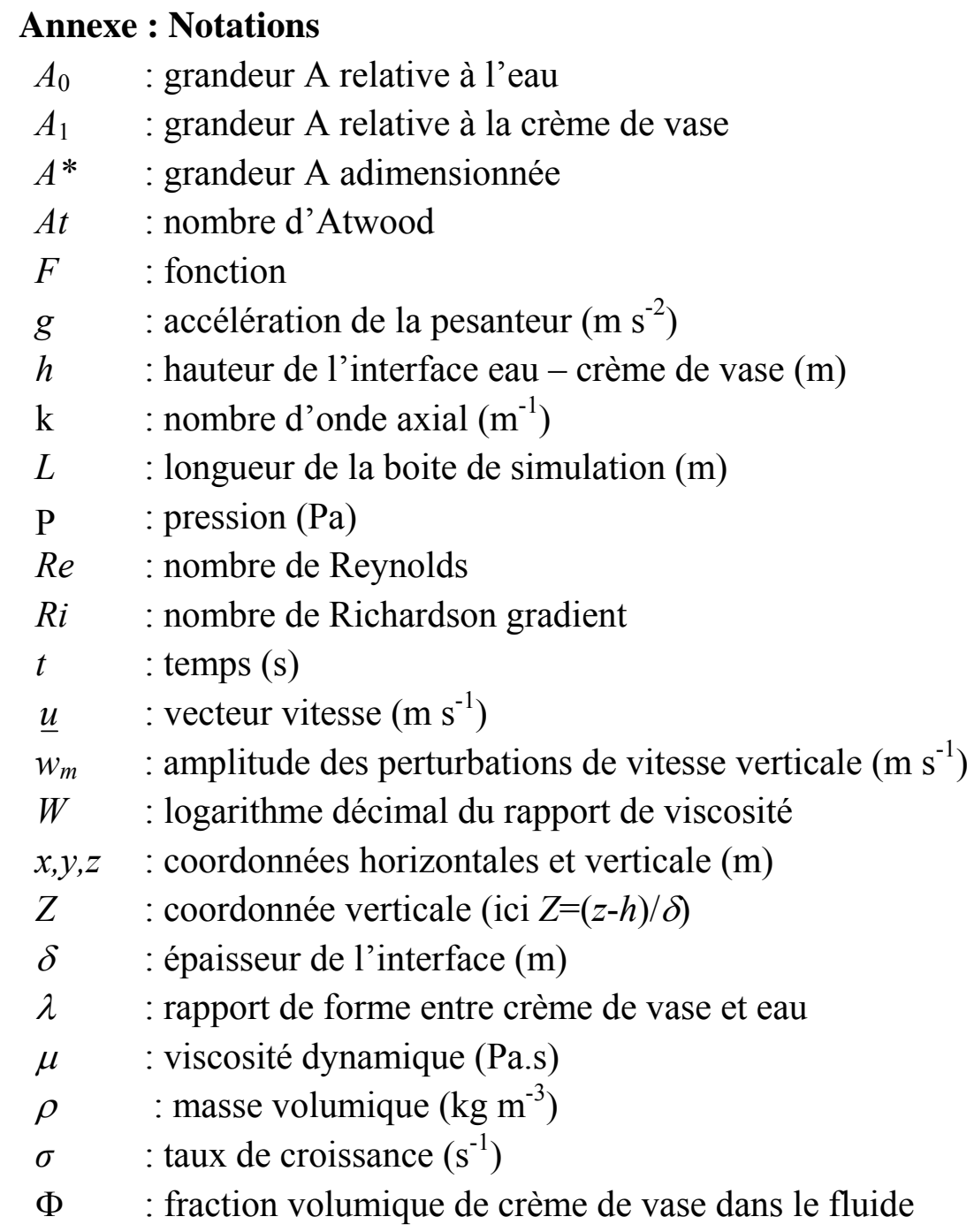

